\title{
Quantitäten, Qualitäten und Kostenzuordnung von Leistungen in Universitätsrechenzentren
}

\author{
W. Held ${ }^{1}$, J. W. Münch ${ }^{2}$
}

\section{Leistungs- und Kostenrechnung in Nordrhein-Westfalen}

In den Hochschulen Nordrhein-Westfalens wird die Kosten- und Leistungsrechnung zum 01.01.2001 verbindlich eingeführt. Ihre verstärkte Diskussion führt zur kritischen Betrachtung aller Handlungsräume sowohl in den Fakultäten als auch in den Zentralen Einrichtungen. Wünschenswert wären neben gleichen Kontrollmechanismen auch gleiche Kataloge von Qualitätsmerkmalen und Leistungsmengen für alle Dienste der Universität. Diese werden allerdings auf Grund sehr vieler Unterschiede nicht leicht festzulegen sein. Trotzdem wird die Öffentlichkeit eine weiterreichende Transparenz von Leistung und Qualität fordern, die über das bisher von Universitäten Veröffentlichte hinausgeht. In der Konkurrenzsituation der Hochschulen untereinander und im modischen Trend à la USA, alles nach seinem ,shareholder-value“ zu hinterfragen, wird die einzelne Universität versuchen, ihre betriebswirtschaftliche Situation zu präsentieren und Leistungsbilanzen vorzulegen. Münch et al. [ 7] haben zur Leistungs- und Kostenrechnung grundsätzliche Überlegungen angestellt. Hier soll eine bisher noch fehlende ausführliche Beschreibung der Quantitäten und Qualitäten der Leistungen ergänzt werden.

Sollte eine Umlage der Kosten Zentraler Einrichtungen auf die Fachbereiche in Erwägung gezogen werden, muss man deutlich darauf hinweisen, dass dies allenfalls auf der Basis von groben, beliebig kritisierbaren Schätzungen möglich ist. Auch hierzu werden Vorschläge präsentiert.

\section{Quantitative Leistungsmerkmale}

Leistungen, die quantifizierbar sind, sollten auf ihre Relevanz überprüft werden. Merkmale, die angemessen sind bei der Darstellung der Leistungen eines Universitätsrechenzentrums (URZ), sollten gesammelt und regelmäßig veröffentlicht werden, mindestens einmal im Jahresbericht. Dies bietet den Nutzern Einsicht in die Möglichkeiten der Informationsverarbeitung (IV) und den Gremien Hilfen zur Verabschiedung zweckmäßiger Empfehlungen.

Leistungsdaten etwa eines einzelnen Servers sind dabei in Folge seiner Verkettung mit anderen Servern und der Einbindung in das LAN wenig aussagefähig. Viel wichtiger sind Leistungen, die am Ende beim Nutzer ankommen. Die Leistung ist nur dann befriedigend, wenn z.B. das LAN und die Server-Kette vollständig funktionieren. In der Universität Münster werden verteilt in der Universität - Messpunkte installiert, die nahe beim Nutzer Daten über das Betriebsverhalten der Netze und Server sammeln sollen. Für Server und Netze sind ihre Frequentierung und Belegung sowie Ausfallzeiten und -häufigkeiten von Bedeutung.

Einige Beispiele von quantifizierbaren Leistungen (Mengen und Zeitangaben) sind: Antwortzeiten der Rechner für den Nutzer, Auslastung der Batch-Systeme und Anzahl ihrer Nutzer-

\footnotetext{
${ }^{1}$ Universität Münster, Zentrum für Informationsverarbeitung (Universitätsrechenzentrum), Münster

${ }^{2}$ Universität-GH Siegen, Hochschulrechenzentrum, Siegen
} 
gruppen, Anzahl der Dialognutzer, Fileserver-Kapazitäten, Anzahl und Kapazitäten im Backup- und Archivierungssystem, Mengenangaben zur Drucker- und Plotternutzung, Betriebsverhalten des Internetzuganges mit dem WWW und den News-Konferenzen, Anzahl der eingehenden und abgehenden E-Mails, Belegung der Wähleingänge und Auslastung des BWINAnschlusses. Außerdem gehören dazu beispielsweise Angaben über die Anzahl der Nutzer und der Nutzer-Beratungen oder die Frequentierung von Lehrveranstaltungen. Auch Öffnungs- und Betriebszeiten des URZ sind hier aufzuführen.

\section{Qualitätsstandards für Leistungen}

Qualität ist die allgemeine Bezeichnung für die Beschaffenheit, Eigenschaft oder Güte einer Sache. Im Gegensatz zur Quantität ist Qualität nicht messbar und nicht zahlenmäßig erfassbar [11]. Zu unterschiedlichen Sichtweisen zum Qualitätsbegriff siehe auch [ 4]. Nach ISO 8402 [ 8] ist Qualität die Gesamtheit von Eigenschaften und Merkmalen eines Produktes oder einer Dienstleistung, die sich auf deren Eignung zur Einführung festgelegter oder vorausgesetzter Erfordernisse beziehen. Bezogen auf die Dienstleistung einer Universität ist Qualität die Beschaffenheit, die eine Dienstleistung zur Erfüllung vorgesehener Forderungen geeignet erscheinen lässt [ 9]. Mit diesen Definitionen müssen für den Dienstleistungserbringer URZ und zum Nutzen seiner Dienstleistungsabnehmer (Mitglieder der Hochschule) Qualitätsmerkmale für die Dienstleistungen festgelegt werden.

Aus Sicht der URZ müssen Kriterien für die Erfassung der „Erfüllung von subjektiven und objektiven Anforderungen und Erwartungen der Kunden“ (www.kconsult.de) gefunden werden. Diese Kriterien müssen im Voraus festgelegt werden, damit Qualität anschließend gesichert und überprüft werden kann. Diese Kriterien sind variabel, denn Qualität ist relativ und sollte möglichst immer weiter verbessert und angepasst werden. Sind diese Kriterien definiert, muss ebenso notwendig eine Qualitätssicherung erfolgen. „Qualitätssicherung umfasst alle diejenigen geplanten und systematischen Tätigkeiten, die notwendig sind, um beim Kunden ein hinreichendes Vertrauen zu schaffen, dass eine Leistung auch die vorher festgelegten Qualitätsanforderungen erfüllt“.

Ausgangsbasis zur Festlegung der Qualitäten (wie auch der Quantitäten) ist der Dienstleistungskatalog des URZ. In diesem muss zu jeder Dienstleistung die angestrebte Qualität so festgelegt sein, dass sie vor allem die Erwartungen der Nutzer des URZ erfüllt. Da derartige Dienstleistungskataloge sehr lang sein können, muss man gegebenenfalls Gruppierungen in Leistungsmengen vornehmen. Die Autoren schlagen vor, die Dienstleistungen nach dem in Münch et al. [ 7] genannten Leistungsmengen zu gruppieren. Auch die Anzahl von Qualitätskriterien muss übersichtlich und daher möglichst klein bleiben. Mehr als 15 derartige Qualitätskriterien dürften kaum zuzuordnen sein.

Die URZ sollten möglichst schon ohne den aufwendigen Aufbau von Mechanismen zur Qualitätssicherung nach ISO 9000 [ 2] zu einem Qualitätsmanagement kommen. Sie sollten eine/n leitende/n Mitarbeiter/in als Qualitätsbeauftragte/n bestimmen, der/die sich um die Umsetzung zu kümmern hat. Die Festlegung der Qualitäten der einzelnen Dienste sollte in Abstimmung mit den Mitarbeiter/innen geschehen. Infolge der Denkanstöße durch Mitarbeiter/innen werden sich beiläufig gleich eine Reihe von Diensten verbessern lassen: Man muss einfach einmal Zeit finden, über das eigene Tun nachzudenken. Bei der Festsetzung von Qualitätskriterien kann man unterscheiden zwischen denen der eingesetzten Produkte und denen der angebotenen Dienste. 


\subsection{Qualitätskriterien für im URZ eingesetzte Produkte}

Für Soft- und Hardwareprodukte kann das URZ keine Qualitätsgarantie z.B. in Bezug auf Korrektheit, Zuverlässigkeit, Aktualität, Vollständigkeit, Flexibilität, Sicherheit, Benutzerfreundlichkeit übernehmen, da es diese selbst nicht herstellt, sondern nur aus einem Spektrum von Möglichkeiten aussucht. Insofern trifft hier der Qualitätsbegriff das Rechenzentrum nur bedingt. Es kann aber - und darin sollte die Qualität seiner Kompetenz liegen - auf die Mängel von Hard- und Software hinweisen. Insbesondere kann es vor der Beschaffung von Produkten Qualitätsmerkmale festlegen und überprüfen und sich somit bemühen, Produkte zu beschaffen, die den erforderlichen Merkmalen entsprechen.

Bei der Qualitätsbetrachtung des Betriebes der Hardware liegen sowohl eine möglichst hohe Ausfallsicherheit und die Planung einer rechtzeitigen Kapazitätsanpassung als auch die Auswahl, die den Benutzeranforderungen adäquat ist und Engpässe nicht entstehen lässt, im Verantwortungsbereich der URZ. Die Softwarequalität vieler Produkte lässt oftmals zu wünschen übrig. Hier kann das URZ im Wesentlichen Erfahrungen sammeln und diese in der Beratung oder in Dokumentationen weitergeben. Es wird auch engen Kontakt zu den Herstellern zu halten versuchen, um rechtzeitig Fehlerhinweise und (Zwischen-)Lösungen zu erhalten, um sie in Form sogenannter Patches an seine Kunden weiterzugeben.

\subsection{Qualitätskriterien für Dienste des URZ}

Qualitätsmerkmale für Dienste beziehen sich auf die Arbeitsprozesse der Mitarbeiter/innen. Dies können z. B. die Folgenden (Tabelle 1) sein:

1. Fachkompetenz:

- Qualität und Vollständigkeit von Informationen, Beratungen und Auskünfte

- Fachwissen und Kenntnisse

- Entscheidungsfähigkeit, Flexibilität

- Erkennung und Beachtung eigener Grenzen

2. Transparenz der Dienstabläufe

- Struktur und Organisation des URZ

- Systematik der Verfahren und Ergebnisse

3. Zuverlässigkeit und Sicherheit

- Zusagen, Absprachen und Termine

- Gleichbehandlung vergleichbarer Fälle

- Widerspruchsfreie Entscheidungen und Auskünfte

- Sicherheit der Dienste und des Betriebes

4. Bearbeitungszeit und Aktualität

- Zügige Bearbeitung

- Angemessene Zeit

- Aktualität

\section{Tabelle 1: Qualitätskriterien}

Zur Zuordnung von Qualitäten zu Diensten legen wir die Zusammenfassung dieser Dienste in Leistungsmengen nach Münch et al. [ 7] zu Grunde (Tabelle 2 bis Tabelle 6).

\begin{tabular}{|c|l|l|}
\hline \multicolumn{2}{|l|}{ Kommunikationssysteme } & Qualitäten \\
\hline Dienste/Prozesse & Quantitäten & $\begin{array}{l}\text { Sicherheit der Dienste und des } \\
\text { Betriebes }\end{array}$ \\
\hline WIN-Anschluss & $\begin{array}{l}\text { Auslastung } \\
\text { Ausfallzeiten, Verfügbarkeit } \\
\text { Antwortzeiten }\end{array}$ & $\begin{array}{l}\text { Sicherheit der Dienste und des } \\
\text { Betriebes }\end{array}$ \\
\hline Wähl-/Standleitungen & $\begin{array}{l}\text { Auslastung, Belegung } \\
\text { Ausfallzeiten }\end{array}$ & $\begin{array}{l}\text { Sicherheit der Dienste und des } \\
\text { Betriebes }\end{array}$ \\
\hline LAN-Backbone/Anschlüsse & $\begin{array}{l}\text { Vorhandene Anschlusspunkte } \\
\text { Vorhandene Kapazitäten }\end{array}$ \\
\hline
\end{tabular}

5. Erreichbarkeit der Dienstleistenden

Zuständigkeiten

- Erreichbarkeit und Vertretungen (z.B. zentraler Anlaufpunkt)

6. Soziale Kompetenz

- Hilfsbereitschaft, partnerschaftlicher Umgang

- Ernstnahme der Kunden

- Offener Umgang mit Konflikten

7. Innovation

- Sicherheit bei der Bewertung neuer Entwicklungen

- Nachhaltigkeit bei der Einführung neuer Entwicklungen

8. Erscheinungsbild des räumlichen Umfeldes

- Dienstgebäude

- Eingangsbereich und Flure

- Arbeits- und Besprechungszimmer

- Sauberkeit und Ordnung 


\begin{tabular}{|c|l|l|}
\hline & Ausfallzeiten & Aktualität \\
\hline Planung und Aufbau & $\begin{array}{l}\text { Warteschlange für neue Anschlüsse } \\
\text { oder Anschlussänderungen }\end{array}$ & $\begin{array}{l}\text { Entscheidungsähigkeit, Flexibilität } \\
\text { Gleichbehandlung vergleichbarer } \\
\text { Fälle } \\
\text { Angemessene Zeit }\end{array}$ \\
\hline Netzberatung & Aufwand in Stunden & $\begin{array}{l}\text { Qualität und Vollständigkeit von } \\
\text { Informationen, Beratungen und } \\
\text { Auskünften } \\
\text { Transparenz } \\
\text { Zügige Bearbeitung } \\
\text { Erreichbarkeit und Vertretungen } \\
\text { (z.B. zentraler Anlaufpunkt) } \\
\text { Soziale Kompetenz }\end{array}$ \\
\hline Weiterentwicklungen & $\begin{array}{l}\text { Sicherheit bei der Bewertung neuer } \\
\text { Entwicklungen } \\
\text { Nachhaltigkeit bei der Einführung } \\
\text { neuer Entwicklungen }\end{array}$ \\
\hline
\end{tabular}

Tabelle 2: Quantitäten und Qualitäten für Kommunikationssysteme

\begin{tabular}{|c|c|c|}
\hline \multicolumn{3}{|c|}{ Rechner u. Betriebssysteme } \\
\hline Dienste/Prozesse & Quantitäten & Qualitäten \\
\hline $\begin{array}{l}\text { Compute-, Dialog-, E-Mail-, } \\
\text { WWW-Server, Server für } \\
\text { Backup, Archivierung und } \\
\text { Sonstige }\end{array}$ & $\begin{array}{l}\text { Auslastung, Belegung, Kapazitäten } \\
\text { Anzahl Nutzer, Gruppen } \\
\text { Ausfallzeiten, } \\
\text { Antwort- oder Reaktionszeiten }\end{array}$ & $\begin{array}{l}\text { Sicherheit der Dienste und des Be- } \\
\text { triebes } \\
\text { Zügige Bearbeitung } \\
\text { Angemessene Zeit }\end{array}$ \\
\hline $\begin{array}{l}\text { Drucker, Plotter und grafi- } \\
\text { sche Ausgabe }\end{array}$ & $\begin{array}{l}\text { Druckerseiten, Plots } \\
\text { Ausfallzeiten }\end{array}$ & $\begin{array}{l}\text { Sicherheit der Dienste und des Be- } \\
\text { triebes } \\
\text { Zügige Bearbeitung }\end{array}$ \\
\hline Eingabe-Peripherie & Ausfallzeiten & $\begin{array}{l}\text { Sicherheit der Dienste und des Be- } \\
\text { triebes }\end{array}$ \\
\hline CIP-Pools im URZ & $\begin{array}{l}\text { Auslastung, Belegung } \\
\text { Anzahl Nutzer, Gruppen } \\
\text { Vorhandene Kapazitäten } \\
\text { Ausfallzeiten } \\
\end{array}$ & $\begin{array}{l}\text { Sicherheit der Dienste und des Be- } \\
\text { triebes } \\
\text { Funktionalitäten }\end{array}$ \\
\hline Nutzer-PCs und -WS & $\begin{array}{l}\text { Auslastung, Belegung } \\
\text { Anzahl Nutzer, Gruppen } \\
\text { Vorhandene Kapazitäten } \\
\text { Ausfallzeiten } \\
\end{array}$ & $\begin{array}{l}\text { Sicherheit der Dienste und des Be- } \\
\text { triebes } \\
\text { Zügige Bearbeitung }\end{array}$ \\
\hline Systemmanagement & $\begin{array}{l}\text { Umfang der überwachten Ressour- } \\
\text { cen }\end{array}$ & $\begin{array}{l}\text { Sicherheit der Dienste und des Be- } \\
\text { triebes } \\
\text { Zuverlässigkeit SW } \\
\text { Aktualität } \\
\text { Funktionalitäten }\end{array}$ \\
\hline PC-Reparatur & Aufwand in Stunden & $\begin{array}{l}\text { Zusagen, Absprachen und Termine } \\
\text { Umgangsformen, Ernstnahme der } \\
\text { Kunden }\end{array}$ \\
\hline Beratung & Aufwand in Stunden & $\begin{array}{l}\text { Qualität und Vollständigkeit von } \\
\text { Informationen, Beratungen und } \\
\text { Auskünfte } \\
\text { Fachwissen und Kenntnisse der } \\
\text { Organisation } \\
\text { Erkennung und Beachtung eigener } \\
\text { Grenzen } \\
\text { Zusagen, Absprachen und Termine } \\
\text { Erreichbarkeit und Vertretungen } \\
\text { (z.B. zentraler Anlaufpunkt) } \\
\text { Hilfsbereitschaft, partnerschaftli- } \\
\text { cher Umgang } \\
\text { Ernstnahme der Kunden }\end{array}$ \\
\hline
\end{tabular}




\begin{tabular}{|l|l|l|}
\hline $\begin{array}{l}\text { Verkauf Software, } \\
\text { Verbrauchsmaterial }\end{array}$ & Anzahl der Vorgänge und Umsatz & $\begin{array}{l}\text { Zügige Bearbeitung } \\
\text { Aktualität } \\
\text { Umgangsformen, Ernstnahme der } \\
\text { Kunden }\end{array}$ \\
\hline Weiterentwicklungen & Aufwand in Stunden & $\begin{array}{l}\text { Innovation } \\
\text { Sicherheit bei der Bewertung neuer } \\
\text { Entwicklungen } \\
\text { Nachhaltigkeit bei der Einführung } \\
\text { neuer Entwicklungen }\end{array}$ \\
\hline
\end{tabular}

Tabelle 3: Quantitäten und Qualitäten für Rechner- und Betriebssysteme

\begin{tabular}{|l|l|l|}
\hline Anwendungen, Informationssysteme & Qualitäten \\
\hline Dienste/Prozesse & Quantitäten & $\begin{array}{l}\text { Zuverlässigkeit SW } \\
\text { Sicherheit } \\
\text { Aktualität, Versionsstände } \\
\text { Benutzerfreundlichkeit } \\
\text { Informationsdienste } \\
\text { Funktionalitäten }\end{array}$ \\
\hline $\begin{array}{l}\text { Softwaremanagement, } \\
\text { Beratung }\end{array}$ & Aufwand in Stunden & $\begin{array}{l}\text { Qualität und Vollständigkeit von } \\
\text { Informationen, Beratungen und } \\
\text { Auskünfte der } \\
\text { Fachwissen und Kenntnisse der } \\
\text { Organisation } \\
\text { Erkennung und Beachtung eigener } \\
\text { Grenzen } \\
\text { Zusagen, Absprachen und Termine } \\
\text { Erreichbarkeit und Vertretungen } \\
\text { (z.B. zentraler Anlaufpunkt) } \\
\text { Hilfsbereitschaft, partnerschaftli- } \\
\text { cher Umgang } \\
\text { Ernstnahme der Kunden }\end{array}$ \\
\hline Weiterentwicklungen & Aufwand in Stunden & $\begin{array}{l}\text { Sicherheit bei der Bewertung neuer } \\
\text { Entwicklungen } \\
\text { Nachhaltigkeit bei der Einführung } \\
\text { neuer Entwicklungen }\end{array}$ \\
\hline
\end{tabular}

Tabelle 4: Quantitäten und Qualitäten für Anwendungen und Informationssysteme

\begin{tabular}{|c|c|c|}
\hline \multicolumn{3}{|l|}{ Ausbildung } \\
\hline Dienste/Prozesse & Quantitäten & Qualitäten \\
\hline $\begin{array}{l}\text { Textverarbeitung, Program- } \\
\text { miersprachen, Kommunika- } \\
\text { tions- und Informationsdiens- } \\
\text { te, Statistik und Numerik } \\
\text { Grafische Anwendungen, Da- } \\
\text { tenbanken, Multimedia, } \\
\text { UNIX, Windows NT sowie } \\
\text { Sonstige Kurse }\end{array}$ & $\begin{array}{l}\text { Anzahl der Ausgebildeten } \\
\text { Anzahl der Kurse } \\
\text { Vielfalt der Themen }\end{array}$ & 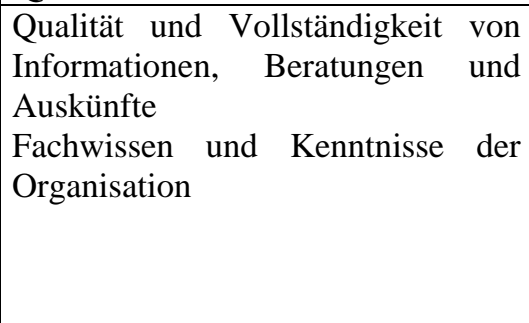 \\
\hline
\end{tabular}

Tabelle 5: Quantitäten und Qualitäten für die Ausbildung

\begin{tabular}{|l|l|l|}
\hline Leitung & & \\
\hline Dienste/Prozesse & Quantitäten & Qualitäten \\
\hline $\begin{array}{l}\text { Personal-, Ressourcen-, Fi- } \\
\text { nanzmanagement, Strategi- } \\
\text { sche Planungen }\end{array}$ & Aufwand in Stunden & $\begin{array}{l}\text { Entscheidungsfähigkeit, Flexibilität } \\
\text { Erkennung und Beachtung eigener } \\
\text { Grenzen } \\
\text { Gleichbehandlung vergleichbarer } \\
\text { Fälle }\end{array}$ \\
\hline
\end{tabular}




\begin{tabular}{|c|c|c|}
\hline & & $\begin{array}{l}\text { Widerspruchsfreie Entscheidungen } \\
\text { und Auskünfte } \\
\text { Erreichbarkeit und Vertretungen } \\
\text { (z.B. zentraler Anlaufpunkt) } \\
\text { Hilfsbereitschaft, partnerschaftli- } \\
\text { cher Umgang } \\
\text { Offener Umgang mit Konflikten } \\
\text { Erscheinungsbild des Dienstgebäu- } \\
\text { des } \\
\text { Eingangsbereich und Flure } \\
\text { Arbeits- und Besprechungszimmer } \\
\text { Sauberkeit und Ordnung } \\
\text { Sicherheit bei der Bewertung neuer } \\
\text { Entwicklungen } \\
\text { Nachhaltigkeit bei der Einführung } \\
\text { neuer Entwicklungen }\end{array}$ \\
\hline $\begin{array}{l}\text { Rektorat und Gremien, } \\
\text { Technologietransfer }\end{array}$ & Aufwand in Stunden & $\begin{array}{l}\text { Qualität und Vollständigkeit von } \\
\text { Informationen, Beratungen und } \\
\text { Auskünfte } \\
\text { Entscheidungsfähigkeit, Flexibilität } \\
\text { Erkennung und Beachtung eigener } \\
\text { Grenzen } \\
\text { Gleichbehandlung vergleichbarer } \\
\text { Fälle } \\
\text { Widerspruchsfreie Entscheidungen } \\
\text { und Auskünfte } \\
\text { Fachwissen und Kenntnisse der } \\
\text { Organisation } \\
\text { Zusagen, Absprachen und Termine } \\
\text { Offener Umgang mit Konflikten }\end{array}$ \\
\hline
\end{tabular}

Tabelle 6: Quantitäten und Qualitäten für die Leitungsfunktionen

Diese Zuordnung von Quantitäten und Qualitäten zu den Diensten eines URZ stellt eine erste Version dar. Die Autoren freuen sich über jede Diskussion zur Weiterentwicklung dieser Tabellen.

\section{Kontrolle von Qualitätsstandards}

Qualitätsmerkmale von Leistungen lassen sich leider nur schwer überprüfen. Dennoch kann auf eine Überprüfung nicht verzichtet werden. Für Verletzungen der Standards sind Eskalationsverfahren vorzusehen. Betriebe gehen teilweise zu einem Total Quality Management (TQM) über, um ihren Geschäftserfolg dauerhaft zu sichern, zufriedene Kunden und Mitarbeiter/innen zu haben und um zu einem positiven Image zu kommen. Von der Einführung und Sicherung eines TQM leben viele Berater. Qualitätspreise werden ausgelobt (z. B. European Quality Award). In Japan gibt es einen vergleichbaren Preis übrigens schon seit 1951. TQM ist zunächst ein Stichwort. Über TQM allein aber werden die notwendigen Verfahren für ein URZ nicht bereitgestellt. TQM macht eher vage Angaben über die Vorgehensweise zur ständigen Verbesserung der Qualität. So werden die Kundenorientierung, ganzheitliches Denken und unmittelbare Verantwortung der Mitarbeiter/innen hervorgehoben, aber nicht sehr deutlich beschrieben. Qualitätszirkel werden eingeführt. Zielvorgaben für Mitarbeiter/innen beschränken sich auf das „Was“. Das „Wie“ müssen die Mitarbeiter/innen selbständig lösen. TQM scheint für URZ nach dem ersten Eindruck ein zu hoch gegriffenes Instrument zu sein, dessen konkrete Umsetzung unklar bleibt. Allein die mit den Fachbereichen zu verabredenden Dienstleistungs-Grade (Service Level Agreements) sind kaum festzulegen. Die URZ sollten deshalb zur Qualitätssicherung bescheidener mit folgenden Methoden beginnen: 


\subsection{Befragungen}

Befragen sollte man viele Beteiligte und Betroffene, z.B. die Universitätsleitung, die zuständigen Gremien, die Hochschullehrer/innen, die wissenschaftlichen Mitarbeiter/innen und die Studierenden. Befragungen werden von der Zielgruppe abhängen, sie können mehr oder weniger umfangreich sein, in größeren Abständen oder regelmäßig erfolgen. Üblicherweise bieten sich dafür WWW-Seiten oder die Papierform an. Wenige Bewertungen mit „gut", „befriedigend" und „unzureichend" genügen oftmals.

\subsection{Fragebögen zum Selbsttest}

Der Leiter eines URZ, die Abteilungsleiter/innen und die Mitarbeiter/innen sollten Fragebögen entwickeln, die sie in Abständen selbst beantworten. Die Erkenntnisse, die sich dabei bei ehrlicher Beantwortung ergeben, sind womöglich hilfreicher als alle anderen Methoden. Sie umfassen die o.a. angesprochenen Quantitäten und Qualitäten, gehen aber darüber hinaus. Nach McLure und Lopato [ 6] sollten diese Fragen z.B. die folgenden Bereiche umfassen (Tabelle 7):

1. Planung (unter Einbeziehung der Nutzer und 4. Produkte und Dienste Universitätsleitung)

- Langfristige und jährliche Konzepte für Hardware, Software und Netzausstattung

- Vorbereitung und Test von Notfallmaßnahmen

- Projektplanungen

2. Politik und Prozeduren

- Kunden-Service

- Festlegung einer Diensteliste

- Jahresberichte

- Prioritätenregelungen für Dienste

- Ausstattungsstandards

- Sicherheit der IV

- Trouble Ticket System

- Transparenz der Zuständigkeiten

- Information der Nutzer in Fehlerfällen

- Betriebsübersichten über alle Betriebskomponenten

3. Einrichtungen und Mitarbeiter/innen

- Nutzerzugang zu Einrichtungen und Mitarbeiter/innen

- Nutzerinformation, insbesondere Erstnutzer

- Reports zur Inanspruchnahme von Ressourcen

- Messungen als Grundlage für Kapazitätsplanungen

- Modernität vorhandener Ressourcen

- Aktivitäten zur Weiterentwicklung der IV

- Ausreichendes Wissen und hinreichende Erfahrungen der Mitarbeiter/innen

- Eigene Kurse

- Verständnis für Nutzerbedürfnisse

- Verstehen der Nutzerprobleme

- Kommunikationsfähigkeit

- Weiterbildung

- Umsetzung neuer Ideen

- Studentische Hilfskräfte, Dauer ihrer Zugehörigkeit zum URZ

Tabelle 7: Fragebogen in Anlehnung an McLure und Lopato
- Organisationsstruktur (z. B. zentral, dezentral)

- Flächendeckung des LAN

- Spektrum der Anwendungssoftware

- Termintreue

- Nutzerausbildung

- Öffentlichkeitsarbeit

5. Organisation

- Adäquate Organisation

- Verantwortung der Universitätsleitung für die IV

- Zugang des URZ-Leiters zur Universitätsleitung

- Einbindung des URZ in Entscheidungen zur IV

- Wirkungsvolle Kommission für IV

- Nutzergruppen

- Sicherheit der Daten

- Organisation der dezentralen IV-Unterstützung

- Befriedigung der Bedarfe der Nutzer

- Kommunikationswege in der Universität

- Anerkennung des URZ in der Universität

6. Haushaltsmittel

- Kostentransparenz für Nutzer

- Wahlmöglichkeit der Nutzer für effiziente Verwendung der Haushaltsmittel

- Drittmittel

- Ersatz- und Abschreibungsverfahren

- Einnahmemöglichkeiten für Mittel

- Outsourcing-Überlegungen

7. Gebäude

- Sicherheit

- $\quad$ Äußerer und innerer Zustand 


\subsection{Evaluationen}

Mit Befragungen und Selbsttests kann man erste Qualitätssicherungen vornehmen. Mit einer Evaluationen durch Kollegen kann man dies wirkungsvoll ergänzen. Diese FremdEvaluationen sind dann wirksam, wenn die Erkenntnisse vertraulich an das nutzende URZ weitergegeben werden. Nur dann kann man erwarten, dass die Evaluierer offen sprechen und dass die zu untersuchenden URZ bereitwillig an der Evaluation mitwirken. Da Kollegen aus anderen Rechenzentren besonders sachkundig sind, dürften über diese Art der Evaluation die besten Ergebnisse erzielbar sein. Evaluationen durch Beraterfirmen sind meistens relativ teuer und, wie vielfältige Erfahrungen in verschiedenen Hochschulen gezeigt haben, oft wenig inhaltsreich, wenn nicht gar oberflächlich und fehlerhaft.

\section{Kostenzuordnung zu Kostenträgern}

\subsection{Individuelle Abrechnung der Dienste}

Betriebswirtschaftlich denkende Wissenschaftler schlagen vereinzelt vor, die Angebote eines URZ durch den freien Wettbewerb mit Anbietern außerhalb der Hochschulen entscheiden zu lassen. Sie übersehen dabei allzu oft einige wichtige Randbedingungen:

- Universitäten und ihre Einrichtungen sind keine Wirtschaftsbetriebe, die gewinnorientiert arbeiten sollen. Sie haben andere Ziele. Eine Kostenfestsetzung kann also nicht nach den Gesetzen des Marktes erfolgen. Die Dienstleistungen können nicht beliebig bepreist werden. Berechnet werden können in der Regel nur die verursachten Kosten.

- Eine genaue Kostenfestsetzung ist aber nicht möglich. Die Abrechnungen etwa nach der Inanspruchnahme einzelner Ressourcen (z. B. Rechenzeit, belegte Magnetplatten), die früher einmal üblich war, treffen den Kern nicht mehr. Ihre konsequente Anwendung würde eine sehr große Zahl von Messwerten beanspruchen, die in vielen Fällen gar nicht eindeutig wären. Soll man etwa für Software die Aufrufhäufigkeit oder Aufrufdauer messen, soll man die Leistungsklasse der Rechner berücksichtigen? Werden diese Daten überhaupt bereitgestellt? Welche Querbezüge zu anderen Softwareprodukten bestehen? Oder das Beispiel E-Mail: Will man die Anzahl der empfangenen E-Mails (für die man gar nicht verantwortlich ist) oder die Anzahl der gesendeten zählen? Oder will man die Länge der E-Mails messen oder die Dauer der Lagerung auf dem E-Mail-Server? Kurz: Wenn man in die Einzelheiten der Inanspruchnahme geht, wird die Festlegung der richtigen Messzahl immer aufwendiger. Dabei ist eine Beliebigkeit nicht zu vermeiden.

- Die im URZ eingesetzten Ressourcen und die angebotenen Dienste sind nicht in das Belieben des URZ gestellt. Vielmehr legen Ministerien, der Wissenschaftsrat, die Deutsche Forschungsgemeinschaft (DFG) und verschiedene Hochschulgremien Randbedingungen der IV fest. Dazu gehören z.B. Verteilung investiver und laufender Haushaltsmittel, Personalausstattung und Gebäude.

- Ein URZ, das Forschung und Lehre wirksam unterstützen will, muss neue Technologien und Dienste früh anbieten, obwohl diese gerade in der Anfangszeit oft teuer sind und in der ersten Zeit von sehr Wenigen in Anspruch genommen werden.

- Eine Einzelabrechnung der vielfältigen Dienste erfordert in Folge des damit verbundenen Verwaltungsaufwandes eine eigene Rechnungsabteilung im URZ, die sich in den anderen Einrichtungen der Universität zwecks Bezahlung und Verbuchung widerspiegeln muss.

Deshalb und aus vielen anderen Gründen empfehlen die Autoren das folgende Verfahren zur Zuordnung der Kosten, das einfach ist, den Vorstellungen der einzelnen Universitäten angepasst werden kann und deren Parameter zum Teil auf Empfehlungen der DFG beruhen. Modifikationen im Algorithmus sollten akzeptiert werden, wenn der Verwaltungsaufwand in Grenzen bleibt. Mit einem derartigen Verfahren kann die Transparenz der Kosten erreicht werden. 


\subsection{Vereinfachte Kostenberechnung}

Die im URZ verursachten Kosten sind universitären Bereichen (z.B. Fachbereichen, Zentralen Einrichtungen, Instituten oder anderen Einheiten) angemessen zuzuordnen. Die Berechnung der tatsächlich verursachten Kosten pro Universitätseinheit kann sehr aufwendig und damit in hohem Maße unwirtschaftlich werden. Der Aufwand der Kostenberechnung muss also in einem vertretbaren Verhältnis zu den berechneten Kosten stehen. Daher sollte auf einfach feststellbare Kenngrößen zurückgegriffen werden. Das Verfahren muss fair, plausibel und auch für Nicht-Mathematiker nachvollziehbar sein. Diese Kenngrößen sollten auch für kleinere Einheiten (z.B. Lehrstühle und Institute) existieren, damit bei Bedarf eine feinere Aufschlüsselung möglich ist. Als primäre Kenngrößen bieten sich die Anzahlen

$\mathrm{NW}_{\mathrm{i}}$ der Wissenschaftler,

$\mathrm{NS}_{\mathrm{i}}$ der Studierenden und

$\mathrm{NL}_{\mathrm{i}}$ der Netzanschlusspunkte (des LAN)

in den entsprechenden Bereichen $(\mathrm{i}=1,2, \ldots, \mathrm{n})$ der Universität an.

Konzentrieren wir uns zunächst auf die Wissenschaftler: Legt man die Gesamtkosten KGES des URZ entsprechend der Anzahl der Wissenschaftler in den Bereichen auf die Bereiche um, so lautet die Formel (summiert wird über $\mathrm{j}=1,2, \ldots, \mathrm{n}$ )

$$
K W_{i}=K G E S \times \frac{N W_{i}}{\sum_{j} N W_{j}}
$$

Hätten alle Bereiche gleich viele Wissenschaftler, so hätten sie alle den gleichen Kostenanteil zu tragen. Entsprechend hätten Bereiche mit höheren Personalanteilen höhere Kosten zu übernehmen. Dies ist plausibel aber vermutlich noch zu grob strukturiert.

Da nicht alle Wissenschaftler über eigene Arbeitsplatz-Computer verfügen, sollte man die relative Dichte $\mathrm{RW}_{\mathrm{i}}$ der Computerausstattung der Wissenschaftler berücksichtigen. Damit käme man aus (1) auf die Formel

$$
K W_{i}=K G E S \times \frac{N W_{i} \times R W_{i}}{\sum_{j} N W_{j} \times R W_{j}} \quad(2) .
$$

Mehr Computer pro Wissenschaftler führen wiederum zu einem höheren Kostenanteil des entsprechenden Bereichs. Dazu könnte man die Kenngröße RW $\mathrm{H}_{\mathrm{i}}$ durch Zählung der Rechner in den Bereichen erfassen. Einfacher und als Näherung akzeptabel sollten die von der Deutschen Forschungsgemeinschaft (DFG) [ 1] vorgeschlagenen, mittleren Ausstattungszahlen $\mathrm{RW}_{\text {i }}$ pro Wissenschaftler, z. B. 1 Rechner pro 1,5 Wissenschaftler in den Wirtschafts-, Sozialund Rechtswissenschaften sowie in der Medizin oder 1 Rechner pro Wissenschaftler in den Naturwissenschaften sein. Die absoluten Zahlen dieser Empfehlung sind nicht entscheidend. Von Einfluss sind die relativen Ausstattungsgrade zwischen den Bereichen. Wenn Wirtschafts-, Sozial- und Rechtswissenschaften inzwischen einen Ausstattungsgrad von annähernd 1 Rechner pro Wissenschaftler erreicht haben, so sind die Naturwissenschaften inzwischen bei einem Wert größer 1 angekommen; die DFG-Werte sind also noch zu verwenden. 
Will man weitere Parameter zur Verfeinerung hinzu nehmen, so ergibt sich als Abwandlung von (2) die allgemeine Formel

$$
K W_{i}=K G E S \times \frac{W_{i}}{\sum_{j} w_{j}}
$$

mit der allgemeinen Wichtung $\mathrm{w}_{\mathrm{i}}$.

Als dritte Kenngröße bieten sich z.B. die von der DFG ebenfalls vorgegebenen Kosten (Preise) $\mathrm{PW}_{\mathrm{i}}$ pro Wissenschaftler-Arbeitsplatz sein, z. B. vor einigen Jahren 15.000 DM pro Rechner in den Wirtschafts-, Sozial- und Rechtswissenschaften sowie der Medizin oder 20.000 DM in den Naturwissenschaften. Teurere Rechner sind in der Regel in Verbindung mit vielfältigeren Anwendungen zu sehen und erfordern damit mehr Aufwand und höhere Folgekosten im URZ. Auch hier spielen die absoluten Zahlen natürlich keine Rolle. Vielmehr sind wiederum nur die Relationen von Bedeutung. Die Relationen stimmen auch heute noch, wenn die Rechner jetzt in den unterschiedlichen Bereichen 3.000 bzw. 4.000 DM (15.000:20.000 = 3.000:4.000) kosten.

Für die Wichtung ergibt sich nun aus (3) zusammen mit den Kosten die Form

$$
W_{i}=N W_{i} \times R W_{i} \times P W_{i}
$$

Wenn die Ausstattungszahlen und die Kosten bei den Nicht-Naturwissenschaftlern sich denen der Naturwissenschaftler angepasst haben sollten, wäre es nur plausibel, dass die entsprechenden Bereiche, wie aus der o. a. Formel hervorgeht, einen höheren und die Naturwissenschaften einen kleineren Teil der Gesamtkosten zu tragen hätten. Entsprechend würde sich die Belastung der Naturwissenschaften verringern, wenn die Wissenschaftler der übrigen Bereiche vergleichbar teure Arbeitsplatz-Rechner einsetzen würden wie die Naturwissenschaftler. Auch das wäre fair und plausibel.

Wem die Berücksichtigung allein der Wissenschaftler an der Kostenumlage zu einseitig ist, der kann die Gesamtkosten KGES des URZ in analoger Weise auf die Studierenden der Bereiche i $(\mathrm{i}=1,2, \ldots, \mathrm{n})$ umlegen.

Dazu sind, analog zu (3) und (4) die Formeln

$$
K S_{i}=K G E S \times \frac{S_{i}}{\sum_{j} s_{j}}
$$

mit

$$
w_{i}=N S_{i} \times R S_{i} \times P S_{i}
$$

zu bilden, wobei die relative Ausstattung $\mathrm{RS}_{\mathrm{i}}$ und die Kosten (Preise) $\mathrm{PS}_{\mathrm{i}}$ pro Rechner für die Studierenden ebenfalls von der DFG empfohlen wurden.

Und wem auch dies noch nicht genügt, der kann entsprechend für das lokale Netz verfahren und analog zu (3), (4) und (5), (6) noch

$$
K L_{i}=K G E S \times \frac{l_{i}}{\sum_{j} l_{j}}
$$


mit

$$
l_{i}=N L_{i} \times R L_{i} \times P L_{i} \quad(8)
$$

bilden, wobei $\mathrm{RL}_{\mathrm{i}}$ bzw. $\mathrm{PL}_{\mathrm{i}}$ die relative Dichte der Zahl der Anschlusspunkte (genutzte Anzahl bezogen auf die Zahl der vorhandenen Anschlusspunkte) bzw. die mittleren Kosten pro Anschlusspunkt darstellen. Wem $\mathrm{RL}_{\mathrm{i}}$ und $\mathrm{PL}_{\mathrm{i}} \mathrm{zu}$ kompliziert zu ermitteln sind, setzt diese zunächst vielleicht einfach gleich 1 .

Da man mit $\mathrm{KW}_{\mathrm{i}}, \mathrm{KS}_{\mathrm{i}}$ und $\mathrm{KL}_{\mathrm{i}}$ die Gesamtkosten KGES nunmehr dreimal umgelegt hat, darf man diese an der endgültigen Aufteilung der Kosten natürlich nur anteilig verwenden, in dem man die Kostenanteile $\mathrm{K}_{\mathrm{i}}$ des Universitätsbereichs $\mathrm{i}(\mathrm{i}=1,2, \ldots, \mathrm{n}) \mathrm{zu}$

$$
\begin{aligned}
& K_{i}=\alpha \times K W_{i}+\beta \times K S_{i}+\gamma \times K L_{i} \\
& \text { mit } \alpha+\beta+\gamma=1
\end{aligned}
$$

berechnet.

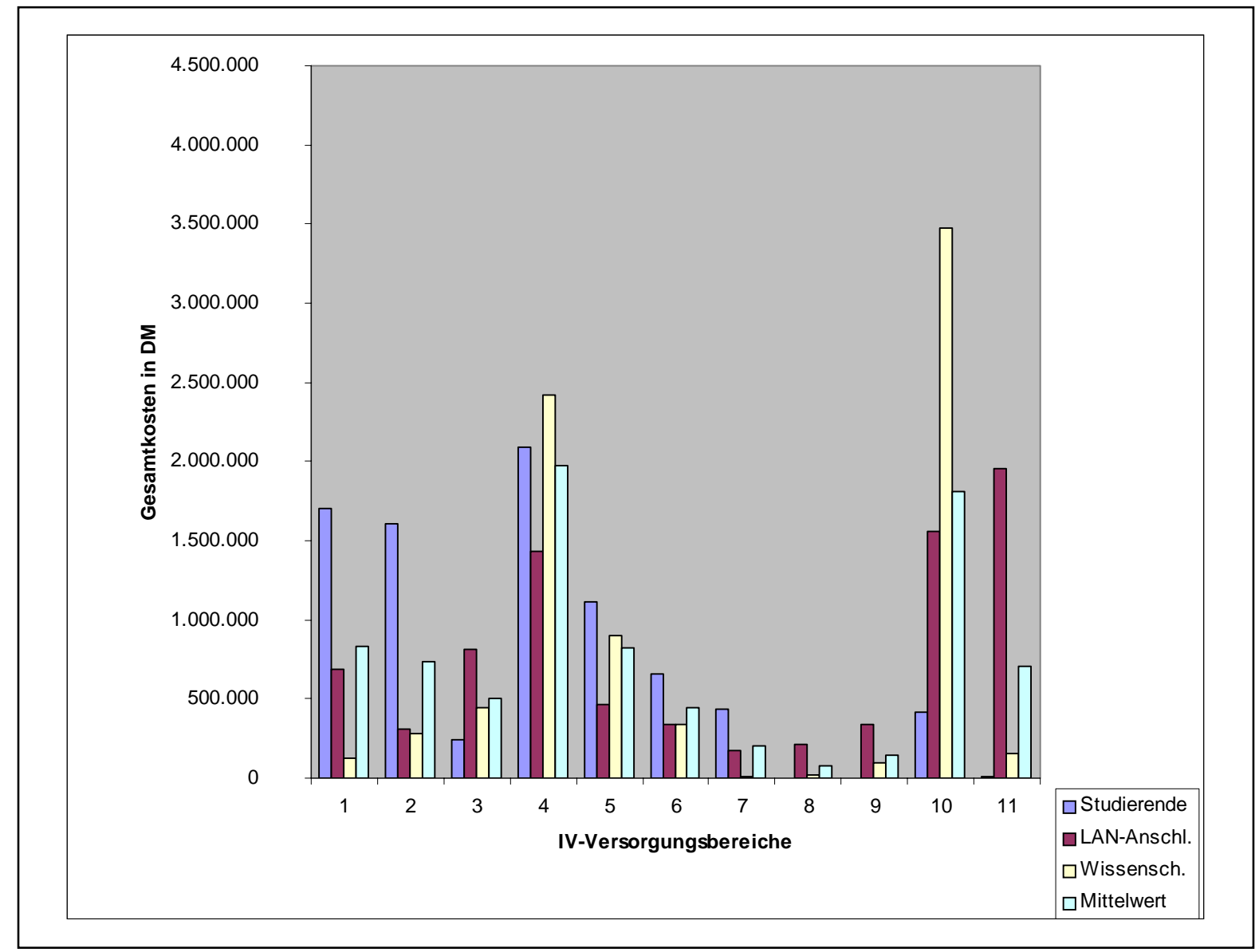

\section{Abbildung 1}

In Abbildung 1 ist eine derartige Kostenverteilung für die IV-Versorgungsbereiche einer Universität beispielhaft aufgeführt. Dabei beruhen die Angaben auf realen Zahlen eines URZ. Die Aufteilung der Gesamtkosten allein auf die Wissenschaftler, Studierenden bzw. Netzan- 
schlusspunkte sind einzeln gezeichnet und dann in einem weiteren Balken mit $\alpha=\beta=\gamma=1 / 3$ als Mittelwert zusammen geführt worden.

Die IV-Versorgungsbereiche entsprechen der Strukturierung in einer Universität. So stehen z.B. die Nummern 2, 4 bzw. 9 für die Versorgungsbereiche Wirtschaftswissenschaften, Naturwissenschaften bzw. Universitätsverwaltung. Die verschiedenen Wichtungen können leicht modifiziert und durch konkrete Rechnungen solange überprüft werden, bis Konsens in der Universität hergestellt worden ist. Natürlich können weitere Einflussfaktoren hinzu genommen werden. Eine Vergrößerung der Zahl der Faktoren wird die Akzeptanz der Umlage allerdings kaum weiter verbessern können. Dieser Algorithmus kann leicht auf die Gesamtkosten, die Kosten der Hauptprozesse oder - wenn erwünscht - auf die der Teilprozesse angewendet werden, etwa in dem von Münch et al. [ 7] vorgeschlagenen Verfahren der Prozesskostenrechnung. Da ein derart einfach zu handhabendes Verfahren auch von kleineren Serviceeinrichtungen der IV, z. B. in Fachbereichen, eingesetzt werden kann, sind schließlich Kostenvergleiche möglich, um zukünftig auch die Organisation der IV-Betreuung immer wieder zu verbessern und anzupassen. Ähnliche Verfahren sind bei anderen Mittelzuweisungen in Universitäten geläufig.

Die Kanzler der Hochschulen in Deutschland haben übrigens in ihrer kürzlich erschienenen Studie zur Kosten- und Leistungsrechnung [ 5] darauf hingewiesen, dass eine exakte Abrechnung der Kosten in Universitäten nicht zweckmäßig ist.

\section{Keine Kostenrechnung ohne Leistungsbeschreibung}

Die Dienstleistungen eines URZ sind umfangreich und komplex. Sie werden für sehr große Nutzerzahlen erbracht. Darüber hinaus unterliegen sie einem schnellen Wandel wie in kaum einem anderen Bereich.

Selbst wenn Qualitäten und Quantitäten der Leistungsmengen des URZ festgelegt, regelmäßig überprüft und eine allgemeine Zustimmung in der Universität finden, wird man es nicht ganz vermeiden können, dass Unzufriedenheit an der einen oder anderen Stelle zum Ausdruck kommt. Entscheidend ist, dass die weit überwiegende Zahl der Nutzer mit ihrem URZ zufrieden ist.

Eine Kostenrechnung sollte grundsätzlich nicht ohne aussagekräftige Leistungsbeschreibung begonnen werden. Wer sich nur auf eine Kostenrechnung beschränkt, handelt leichtfertig, denn sie verleitet zu unvorhersehbaren Eingriffen durch Dritte. Wenn man die Kostenrechnung zur Steuerung und laufenden Verbesserung der Dienste nutzen will - und das ist ein wesentlicher Zweck der Kostenrechnung -, so muss man die Leistungen kennen und genau untersuchen. Die Leistungen und ihre Qualitäten und Quantitäten verursachen die Kosten. Wer die Kosten reduzieren will, der muss schließlich die Frage nach der Reduktion entsprechender Leistungen beantworten.

\section{Literaturhinweise}

[ 1] DFG: Empfehlungen der Kommission für Rechenanlagen, Informationsverarbeitung und Rechner für Hochschulen 1996 bis 2000, Bonn 1998

[ 2] Einführung zu ISO 9000, www.move.muenster.de/4bq-doku/ISO9000.html

[ 3] Hofmann, P., Schmitt, B., IT Service Management, 1998,

www.connector.de/medien/connection/vol16/Conn1198.htm 
[ 4] Jeans, J., Qualität und Quantität, Physik und Philosophie, Zürich 1944

[ 5] Kanzler der Hochschulen der Bundesrepublik Deutschland, Arbeitskreis Hochschulrechnungswesen, www.tu-muenchen.de/aktuell/reformprojekt/AK-HSReW.html

[ 6] McLure, C.R., Lopato, C.L., Assessing the academic networked environments: Strategies and options. Coalition for Networked Information, 1996

[ 7] Münch, W., Held, W., Nelißen, M. und Klein, J., Leistungs- und Kostenrechnung in der Datenverarbeitung am Beispiel der Hochschulrechenzentren in NRW, PIK 21 (1998) 3

[ 8] Qualität nach ISO 8402 , www.move.muenster.de/4bq-doku/WASQUAL.html

[ 9] Rahn,O., Lexikon der Betriebswirtschaftslehrer, Ludwigshafen 1997

[ 10] Sturm, R., Managing Quality of Services, 1997, www.summit.online.com/service/papers/rick1-serv.htm

[11] Universität Hamburg, Psychologie- Fachgebärdenlexikon 